\title{
A Measurement of the Hubble Constant Using Galaxy Redshift Surveys
}

\author{
Yuting Wang ${ }^{1,2}$, Lixin $\mathrm{Xu}^{3}$, and Gong-Bo Zhao ${ }^{1,2}$ \\ ${ }^{1}$ National Astronomy Observatories, Chinese Academy of Science, Beijing, 100012, China; ytwang@ @ao.cas.cn, lxxu@dlut.edu.cn \\ ${ }^{2}$ Institute of Cosmology and Gravitation, University of Portsmouth, Portsmouth, PO1 3FX, UK; gbzhao@ nao.cas.cn \\ ${ }^{3}$ Institute of Theoretical Physics, School of Physics \& Optoelectronic Technology, Dalian University of Technology, Dalian, 116024, China \\ Received 2017 July 3; revised 2017 September 11; accepted 2017 September 20; published 2017 November 6
}

\begin{abstract}
We perform a measurement of the Hubble constant, $H_{0}$, using the latest baryonic acoustic oscillation (BAO) measurements from galaxy surveys of 6dFGS, SDSS DR7 Main Galaxy Sample, BOSS DR12 sample, and eBOSS DR14 quasar sample, in the framework of a flat $\Lambda$ CDM model. Based on the Kullback-Leibler divergence, we examine the consistency of $H_{0}$ values derived from various data sets. We find that our measurement is consistent with that derived from Planck and with the local measurement of $H_{0}$ using the Cepheids and type Ia supernovae. We perform forecasts on $H_{0}$ from future BAO measurements, and find that the uncertainty of $H_{0}$ determined by future BAO data alone, including complete eBOSS, DESI, and Euclid-like, is comparable with that from local measurements.
\end{abstract}

Key words: cosmological parameters - distance scale - large-scale structure of universe

\section{Introduction}

Determining the Hubble constant, $H_{0}$, which is the present expansion rate of the Universe, with high precision plays a crucial role in cosmology, and $H_{0}$ can be measured locally, or derived cosmologically through measurements of the Cosmic Microwave Background (CMB) and Baryon Acoustic Oscillations (BAO; see Freedman \& Madore 2010; Freedman 2017 for a recent review on astronomical methods of $H_{0}$ measurements and their significance in cosmology).

Recently, a direct measurement of $H_{0}$ led by Riess et al. (2016; R16) using Cepheids and type Ia supernovae finds $H_{0}=73.24 \pm 1.74 \mathrm{~km} \mathrm{~s}^{-1} \mathrm{Mpc}^{-1}$, which is a $2.4 \%$ measurement. On the other hand, a recent CMB measurement of $H_{0}$ using the Planck satellite (PLC15) achieved a percent level precision, namely, $H_{0}=67.27 \pm 0.66 \mathrm{~km} \mathrm{~s}^{-1} \mathrm{Mpc}^{-1}$ (Planck Collaboration et al. 2016). Note that, unlike the local measurement, the CMB measurement of the Hubble constant is model-dependent as a cosmological model, which is $\Lambda \mathrm{CDM}$ used for the measurement we quote here, is needed to convert the observed angular diameter distance at $z \sim 1100$ and the sound horizon into a measurement of $\mathrm{H}_{0}$.

These two measurements are in apparent tension at a more than $3 \sigma$ level (Riess et al. 2016). The tension may imply that the $\Lambda \mathrm{CDM}$ used in the CMB analysis needs to be extended (Wyman et al. 2014; Wang et al. 2015; Di Valentino et al. 2016; Pourtsidou \& Tram 2016; Sola et al. 2017; Zhao et al. 2017a), or that the measurements were contaminated by systematics to an unknown level. In this situation, additional independent measurements of $H_{0}$, e.g., using BAO distance measurements derived from galaxy surveys ${ }^{4}$ (Cheng \& Huang 2015), can provide the critical information that we need.

The BAO distance measurements using galaxy redshift surveys play a key role in probing the cosmic expansion history. The BAO characteristic scale can be measured in both radial and transverse directions of the line of sight to provide estimates of the Hubble parameter, $H(z)$, and angular diameter distance, $D_{A}(z)$, respectively, at redshift $z$. Recently,

\footnotetext{
4 There are other methods to determine the Hubble constant using galaxies. See Chen et al. (2017) for an example.
}

the collaboration of the Baryon Oscillation Spectroscopic Survey (BOSS), which is a part of the Sloan Digital Sky Survey (SDSS)-III, performed BAO measurements in the redshift range of $0.2<z<0.75$ using the completed Data Release 12 (DR12; Alam et al. 2017; Wang et al. 2017; Zhao et al. 2017b). The extended BOSS (eBOSS, part of SDSS-IV) detected a BAO signal at a $4 \%$ precision at $z \sim 1.5$ using the DR14 quasar sample (Ata et al. 2017). These new BAO measurements can provide a $H_{0}$ measurement, which is independent of $\mathrm{CMB}$ and local measurements, and thus can be highly informative.

In this paper, we determine the Hubble constant using the BOSS DR12 and eBOSS DR14 BAO measurements, combined with other measurements presently available, and investigate the consistency of $H_{0}$ values derived from different data sets using the Kullback-Leibler (KL) divergence (Kullback \& Leibler 1951). We also perform a forecast for future BAO data for a feasibility study.

This paper is organized as follows. In the next section, we present the method and data used in this work, followed by a section devoted to the results. We present our conclusion and discussions in Section 4.

\section{Method and Data}

In the spatially flat $\Lambda \mathrm{CDM}$ model, the Hubble parameter is

$$
H(z)=H_{0} \sqrt{\Omega_{r}(1+z)^{4}+\Omega_{m}(1+z)^{3}+\Omega_{\Lambda}},
$$

where $\Omega_{r}+\Omega_{m}+\Omega_{\Lambda}=1$. The present energy density of radiation $^{5} \Omega_{r}=\Omega_{m} /\left(1+z_{\mathrm{eq}}\right)$, with $z_{\mathrm{eq}}=2.5 \times 10^{4} \Omega_{m} h^{2}$ $\left(T_{\mathrm{CMB}} / 2.7 \mathrm{~K}\right)^{-4}$ being the redshift of matter-radiation equality. We adopt $T_{\mathrm{CMB}}=2.7255 \mathrm{~K}$. The angular diameter distance is

$$
D_{A}(z)=\frac{1}{1+z} \int_{0}^{z} \frac{d z^{\prime}}{H\left(z^{\prime}\right)} .
$$

\footnotetext{
5 Three species of massless neutrinos are included, and the energy density is given in terms of the photon density, $\rho_{\gamma}$, by $\rho_{\nu}=3(7 / 8)(4 / 11)^{4 / 3} \rho_{\gamma}$.
} 
The sound horizon, $r_{s}$, at the redshift of the drag epoch, $z_{d}$, can be calculated as

$$
r_{d} \equiv r_{s}\left(z_{d}\right)=\int_{0}^{\frac{1}{1+z_{d}}} \frac{d a^{\prime}}{a^{\prime 2} H\left(a^{\prime}\right) \sqrt{3\left(1+\overline{R_{b}} a^{\prime}\right)}},
$$

where $\overline{R_{b}}=3.15 \times 10^{4} \Omega_{b} h^{2}\left(T_{\mathrm{CMB}} / 2.7 \mathrm{~K}\right)^{-4}$. Note that $z_{d}$ is well approximated analytically (Eisenstein \& Hu 1998) as

$$
z_{d}=\frac{1291\left(\Omega_{m} h^{2}\right)^{0.251}}{1+0.659\left(\Omega_{m} h^{2}\right)^{0.828}}\left[1+b_{1}\left(\Omega_{b} h^{2}\right)^{b_{2}}\right],
$$

where

$$
\begin{gathered}
b_{1}=0.31\left(\Omega_{m} h^{2}\right)^{-0.419}\left[1+0.607\left(\Omega_{m} h^{2}\right)^{0.674}\right], \\
b_{2}=0.238\left(\Omega_{m} h^{2}\right)^{0.223} .
\end{gathered}
$$

We use a fixed value of the baryon density $\Omega_{b} h^{2}=0.02225$ from the Planck result (Planck Collaboration et al. 2016). ${ }^{6}$ The baryon density can also be accurately determined in a CMBindependent way, e.g., using the primordial deuterium abundance in the Big Bang Nucleosynthesis (BBN) theory (Riemer-Sørensen \& Sem Jenssen 2017).

Note that the quantities $H(z) r_{d}$ and $D_{A}(z) / r_{d}$ can be estimated from anisotropic BAO measurements, while the quantity,

$$
D_{V}(z) / r_{d} \equiv\left[(1+z)^{2} D_{A}^{2}(z) \frac{z}{H(z)}\right]^{\frac{1}{3}} / r_{d},
$$

is determined by isotropic BAO measurements.

As shown above, the BAO distance measurements, $H(z) r_{d}, D_{A}(z) / r_{d}$ or $D_{V}(z) / r_{d}$, are two-variable functions of $\Omega_{m}$ and $H_{0}$ (once $\Omega_{b} h^{2}$ is known) in a flat $\Lambda$ CDM cosmology, and therefore the Hubble constant can be, in principle, determined from the BAO distances with $\Omega_{m}$ marginalized over.

In what follows, we use isotropic or anisotropic BAO distance measurements to determine the Hubble constant with $\Omega_{m}$ marginalized over, i.e., our parameter space is simply (assuming a flatness of the Universe)

$$
P \equiv\left\{\Omega_{m}, H_{0}\right\} \text {. }
$$

The sound horizon at the drag redshift $r_{d}$ is calculated using Equation (3). ${ }^{7}$ We perform a Monte Carlo Markov Chain (MCMC) global fitting for parameter estimation using a modified version of CosmoMC (Lewis \& Bridle 2002). ${ }^{8}$

The BAO data sets used in this work include

1. the isotropic BAO measurements using the $6 \mathrm{dFRS}(6 \mathrm{dF}$; Beutler et al. 2011) and SDSS main galaxy sample (MGS) (Ross et al. 2015) at effective redshifts $z_{\text {eff }}=0.106$ and $z_{\text {eff }}=0.15$, respectively;

2. the BOSS DR12 anisotropic BAO measurements at three effective redshifts (BOSS 3zbin) in Alam et al. (2017) or at nine effective redshifts (BOSS 9zbin) in Wang et al. (2017) and Zhao et al. (2017b);

\footnotetext{
6 We have tested to marginalize over $\Omega_{b} h^{2}$ with a Gaussian prior derived from the Planck measurement and find that the result is largely unchanged.

7 Except for $6 \mathrm{dF}$, the value of $r_{d}$ is rescaled by a factor $r_{d} / \tilde{r}_{d}$, where $\tilde{r}_{d}$ value is calculated from CAMB (Lewis et al. 2000, available at http://camb.info/) in the same fiducial cosmology (Bennett et al. 2014).

8 http://cosmologist.info/cosmomc/
}

3. the eBOSS DR14 isotropic BAO measurement at $z_{\text {eff }}=1.52$ (Ata et al. 2017); and

4. a combination of $6 \mathrm{dF}+\mathrm{MGS}+$ BOSS 3zbin + eBOSS DR14 (All 3zbin), or a combination of $6 \mathrm{dF}+\mathrm{MGS}+$ BOSS 9zbin + eBOSS DR14 (All 9zbin).

To check the consistency of the $H_{0}$ values determined from different data sets within the $\Lambda \mathrm{CDM}$ model, we compute the tension, $T$, based on the KL divergence (Kunz et al. 2006; Paykari \& Jaffe 2013; Amara \& Refregier 2014; Seehars et al. 2014, 2016; Verde et al. 2014; Grandis et al. 2016; Raveri et al. 2016), which quantifies the distance between two probability density functions (PDFs), $p_{1}$, and $p_{2}$. If both $p_{1}$ and $p_{2}$ are assumed to be Gaussian, the relative entropy in bits between the two PDFs can be evaluated as

$$
\begin{aligned}
D\left(p_{2} \| p_{1}\right)= & \frac{1}{2 \log 2}\left[\operatorname{Tr}\left(\mathcal{C}_{1}^{-1} \mathcal{C}_{2}\right)-d-\log \frac{\operatorname{det} \mathcal{C}_{2}}{\operatorname{det} \mathcal{C}_{1}}\right. \\
& \left.+\left(\theta_{2}-\theta_{1}\right)^{T} \mathcal{C}_{1}^{-1}\left(\theta_{2}-\theta_{1}\right)\right],
\end{aligned}
$$

where $\theta_{i}$ is the best-fit parameter vector, $\mathcal{C}_{i}$ is the corresponding covariance matrix, and $d$ denotes the dimensions of the parameter space (e.g., $d=2$ in our case where both $H_{0}$ and $\Omega_{m}$ are free parameters). If data are assumed to be more informative than the priors, one can compute the expected relative entropy, $\langle D\rangle$, with its standard deviation, $\Sigma$, via

$$
\begin{gathered}
\langle D\rangle \simeq \frac{1}{\log 2}\left[\operatorname{Tr}\left(\mathcal{C}_{2} \mathcal{C}_{1}^{-1}\right)-\frac{1}{2} \log \frac{\operatorname{det} \mathcal{C}_{2}}{\operatorname{det} \mathcal{C}_{1}}\right], \\
\Sigma(D) \simeq \frac{1}{\sqrt{2} \log 2} \sqrt{\operatorname{Tr}\left(\mathcal{C}_{1}^{-1} \mathcal{C}_{2}+\mathbb{I}\right)^{2}}, \\
S \equiv D\left(p_{2} \| p_{1}\right)-\langle D\rangle,
\end{gathered}
$$

where the Surprise, $S$, is defined as the difference between the relative entropy and its expectation value. The tension, $T$, is defined as the signal-to-noise ratio of the Surprise, i.e.,

$$
T \equiv S / \Sigma \text {. }
$$

If $T \lesssim 1$, then $p_{1}$ and $p_{2}$ are consistent with each other, while otherwise the two PDFs are in tension (Seehars et al. 2016).

We also perform forecasts on the uncertainty of $H_{0}$ using ongoing and upcoming redshift surveys, including eBOSS $^{9}$ (Dawson et al. 2016; Zhao et al. 2016), Dark Energy Spectroscopic Instrument (DESI $^{10}$; DESI Collaboration et al. 2016a, 2016b), and ESA's Euclid satellite ${ }^{11}$ (Laureijs et al. 2011). We use a flat, $\Lambda$ CDM cosmology derived from the Planck mission as our fiducial model (Planck Collaboration et al. 2016), take the forecasted BAO data for galaxy surveys of a complete eBOSS from Zhao et al. (2016; i.e., the BAO result from eBOSS luminous red galaxies, high-density emission-line galaxies, and clustering quasars in Table 4 from Zhao et al. 2016), DESI (i.e., the BAO result from DESI luminous red galaxies, emission-line galaxies, and clustering quasars in Table 2.3 from DESI Collaboration et al. (2016a) and DESI bright galaxies in Table 2.5 from DESI Collaboration

\footnotetext{
9 We use eBOSS throughout for the complete 5-year eBOSS sample, while we use eBOSS DR14 to denote the eBOSS DR14 quasar sample. More information on the eBOSS survey is available at http://www.sdss.org/ surveys/eboss/.

${ }^{10}$ http://desi.lbl.gov/

11 http://www.euclid-ec.org/
} 

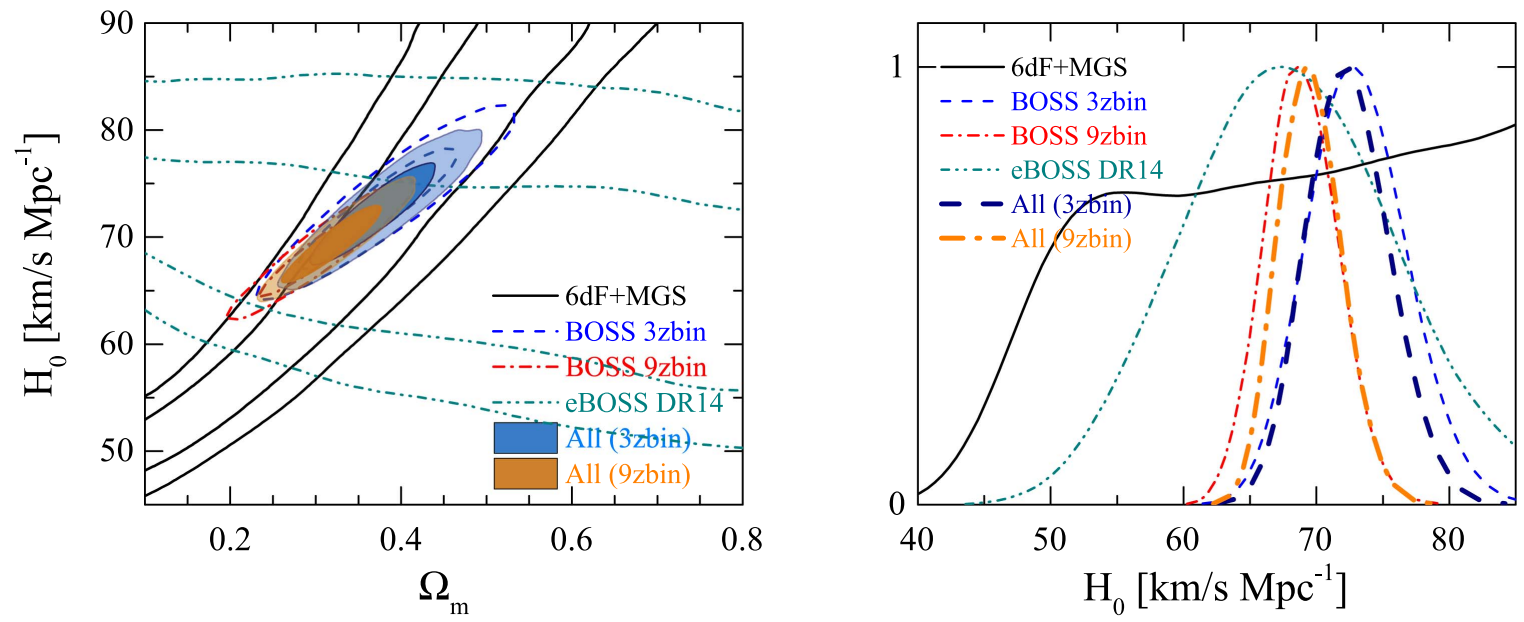

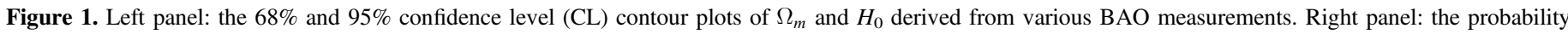
distribution of $H_{0}$ derived from various $\mathrm{BAO}$ measurements.

et al. 2016a), and Euclid-like Font-Ribera et al. (2014; i.e., Table 6 in Font-Ribera et al. 2014), respectively, and perform parameter estimation using the MCMC method, in the same way as we did for the current data sets.

\section{Results}

We present the joint constraint on $H_{0}$ and $\Omega_{m}$, and the posterior probability distribution of $H_{0}$ from various BAO data sets, including the latest eBOSS DR14 quasar sample, in Figure 1. As shown, the contours derived from different data sets show different degeneracy between $H_{0}$ and $\Omega_{m}$. This is expected as the degeneracy is largely determined by the effective redshift at which the BAO measurement is performed. Hence having tomographic BAO measurements at a large number of redshifts helps to break the degeneracy. This can be seen by comparing the "All 3zbin" to the "All 9zbin" results. The only difference in these two data sets is that the BOSS DR12 galaxies were subdivided into more redshift slices in the $9 z$ bin sample to gain more light-cone information. As shown in the upper part of Table 1, the improvement on the uncertainty of $H_{0}$ is significant, namely, the error of $H_{0}$ reduces from 3.05 to $2.34 \mathrm{~km} \mathrm{~s}^{-1} \mathrm{Mpc}^{-1}$, which is a $23 \%$ improvement.

We quantify the (in)consistency among the derived $\Omega_{m}$ and $H_{0}$ from BAO data and PLC15 using the quantity defined in Equation (13). We also calculate the KL divergence between the PDFs for $H_{0}$ with $\Omega_{m}$ marginalized over from various data sets, including those from PLC15 and R16. The result is presented in Table 2, including the relative entropy, $D$, its expected value, $\langle D\rangle$, the Surprise, $S$, in bits, and the tension, $T$, with $1 \sigma$ error. As shown, except for the PLC15 and R16 pair, where $T$ is larger than 1 at about a $2 \sigma$ level, all others are consistent with each other (the tension $T$ are all less than unity).

Given that the best measurement of $H_{0}$ to date using BAO alone (i.e., the "All 9zbin" result) has a worse precision than that from R16 or PLC15, we investigate the constraining capability of future BAO surveys, including the complete eBOSS, DESI, and Euclid-like, on $H_{0}$. The joint constraint on $H_{0}$ and $\Omega_{m}$, and the marginalized constraint on $H_{0}$, from these surveys are shown in Figure 2 and in the lower part of Table 1, respectively. As shown, future galaxy surveys, especially for DESI or Euclid-like alone, are able to provide a better constraint on $H_{0}$ than that of the current CMB constraint, which is promising.
Table 1

The Mean and $68 \%$ CL Constraint on $H_{0}$ Using Various Data Sets

\begin{tabular}{lcc}
\hline \hline Data Set & $H_{0}\left(\mathrm{~km} \mathrm{~s}^{-1} \mathrm{Mpc}^{-1}\right)$ & Precision \\
\hline All 3zbin & $71.75 \pm 3.05$ & $4.25 \%$ \\
All 9zbin & $69.13 \pm 2.34$ & $3.38 \%$ \\
R16 & $73.24 \pm 1.74$ & $2.38 \%$ \\
PLC15 & $67.27 \pm 0.66$ & $0.98 \%$ \\
\hline eBOSS & $67.27 \pm 1.55$ & $2.30 \%$ \\
DESI & $67.27 \pm 0.33$ & $0.49 \%$ \\
Euclid-like & $67.27 \pm 0.21$ & $0.31 \%$ \\
\hline
\end{tabular}

Note. The upper part of the table (above the horizontal line) is for current data sets, while the lower part shows the forecast result based on a fiducial model derived from PLC15.

Table 2

Quantification of the (in) Consistency among Various Data Sets

\begin{tabular}{lrrrrr}
\hline \hline Data Set & $D$ & $\langle D\rangle$ & \multicolumn{1}{c}{$S$} & $T$ & $\sigma_{T}$ \\
\hline 2D: $\left\{\Omega_{m}, H_{0}\right\}$ & & & & & \\
All 3zbin $\rightarrow$ All 9zbin & 0.87 & 2.46 & -1.59 & -0.71 & 0.14 \\
All 3zbin $\rightarrow$ PLC15 & 7.91 & 7.87 & 0.04 & 0.02 & 1.12 \\
All 9zbin $\rightarrow$ PLC15 & 8.58 & 8.31 & 0.27 & 0.09 & 1.54 \\
\hline 1D: $\left\{H_{0}\right\}$ & & & & & \\
All 3zbin $\leftrightarrow$ All 9zbin & 0.62 & 1.23 & -0.61 & -0.38 & 0.19 \\
All 3zbin $\leftrightarrow$ PLC15 & 3.08 & 2.28 & 0.80 & 0.75 & 1.74 \\
All 9zbin $\leftrightarrow$ PLC15 & 1.62 & 1.94 & -0.32 & -0.29 & 0.94 \\
All 3zbin $\leftrightarrow$ R16 & 0.50 & 1.28 & -0.78 & -0.58 & 0.26 \\
All 9zbin $\leftrightarrow$ R16 & 2.33 & 1.23 & 1.10 & 0.70 & 0.42 \\
PLC15 $\leftrightarrow$ R16 & 61.91 & 8.63 & 53.29 & 6.57 & 2.73 \\
\hline
\end{tabular}

Note. Top section: the KL divergence between the PDFs for $\Omega_{\mathrm{m}}$ and $H_{0}$ using BAO data and PLC15. Bottom Section: the KL divergence between the PDFs for $H_{0}$ with $\Omega_{m}$ marginalized over from various data sets. The tension, $T \lesssim 1$, illustrates the relevant pairs of data sets that are consistent with each other.

\section{Conclusion and Discussions}

In this paper, we determine the Hubble constant using BAO measurements from galaxy redshift surveys in a flat $\Lambda \mathrm{CDM}$ cosmology. A combination of recent BAO measurements from 


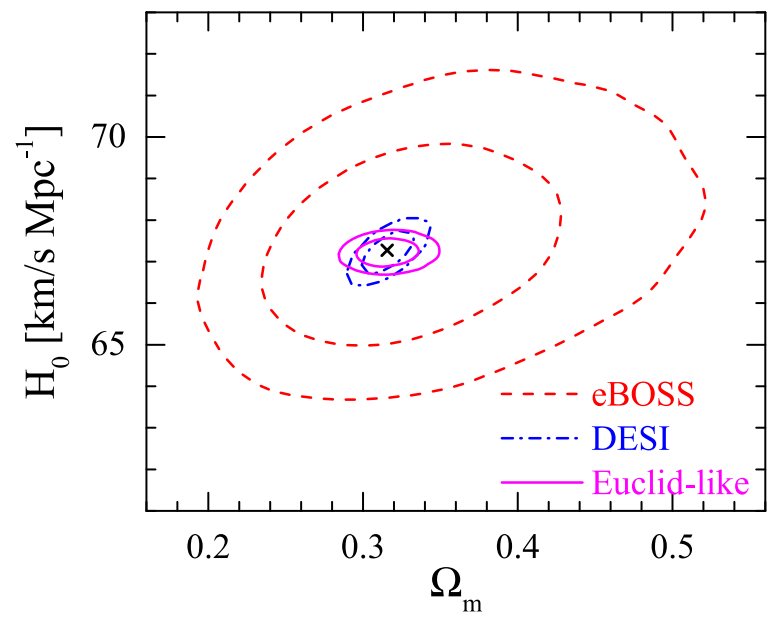

Figure 2. The $68 \%$ and $95 \% \mathrm{CL}$ contour plots of parameters $\Omega_{m}$ and $H_{0}$ in a flat $\Lambda \mathrm{CDM}$ model, derived from the complete eBOSS (red dashed), DESI (blue dash-dotted), and Euclid-like (magenta solid). The black cross corresponds to the fiducial model.

6dF, MGS, BOSS DR12 (with 9 redshift slices), and eBOSS DR14 quasar samples yield a measurement of the Hubble constant, namely, $H_{0}=69.13 \pm 2.34 \mathrm{~km} \mathrm{~s}^{-1} \mathrm{Mpc}^{-1}$, which is a $3.4 \%$ measurement. Given the level of the uncertainty, this measurement is consistent with both R16 and PLC15, which are in tension between themselves.

Based on a forecast, we find that future galaxy surveys, including DESI and Euclid-like, will be able to provide competitive constraints on $H_{0}$, compared with current local or CMB measurements.

We thank Will Percival for discussions and comments. We also thank Chao Liu for discussions. Y.W. is supported by the NSFC grant No. 11403034, and by the Young Researcher Grant of National Astronomical Observatories, Chinese Academy of Sciences. L.X. is supported by the NSFC grant No. 11275035 , Grant No.11675032, and by "the Fundamental Research Funds for the Central Universities" under grant No. DUT16LK31. G.B.Z. is supported by an NSFC grant No. 11673025, and by a Royal Society-Newton Advanced Fellowship.
This research used resources of the SCIAMA cluster supported by University of Portsmouth, and the ZEN cluster supported by NAOC.

\section{References}

Alam, S., Ata, M., Bailey, S., et al. 2017, MNRAS, 470, 2617

Amara, A., \& Refregier, A. 2014, PhRvD, 89, 083501

Ata, M., Baumgarten, F., Bautista, J., et al. 2017, arXiv:1705.06373

Bennett, C. L., Larson, D., Weiland, J. L., \& Hinshaw, G. 2014, ApJ, 794, 135

Beutler, F., Blake, C., Colless, M., et al. 2011, MNRAS, 416, 3017

Chen, Y., Kumar, S., \& Ratra, B. 2017, ApJ, 835, 86

Cheng, C., \& Huang, Q. 2015, SCPMA, 58, 5684

Dawson, K. S., Kneib, J.-P., Percival, W. J., et al. 2016, AJ, 151, 44

DESI Collaboration, Aghamousa, A., Aguilar, J., et al. 2016a, arXiv:1611. 00036

DESI Collaboration, Aghamousa, A., Aguilar, J., et al. 2016b, arXiv:1611. 00037

Di Valentino, E., Melchiorri, A., \& Silk, J. 2016, PhLB, 761, 242

Eisenstein, D. J., \& Hu, W. 1998, ApJ, 496, 605

Font-Ribera, A., McDonald, P., Mostek, N., et al. 2014, JCAP, 5, 023

Freedman, W. L. 2017, NatAs, 1, 0169

Freedman, W. L., \& Madore, B. F. 2010, ARA\&A, 48, 673

Grandis, S., Seehars, S., Refregier, A., Amara, A., \& Nicola, A. 2016, JCAP, 5,034

Kullback, S., \& Leibler, R. A. 1951, Ann. Math. Stat., 22, 79, https:// projecteuclid.org/euclid.aoms/1177729694

Kunz, M., Trotta, R., \& Parkinson, D. R. 2006, PhRvD, 74, 023503

Laureijs, R., Amiaux, J., Arduini, S., et al. 2011, arXiv:1110.3193

Lewis, A., \& Bridle, S. 2002, PhRvD, 66, 103511

Lewis, A., Challinor, A., \& Lasenby, A. 2000, ApJ, 538, 473

Paykari, P., \& Jaffe, A. H. 2013, MNRAS, 433, 3523

Planck Collaboration, Ade, P. A. R., Aghanim, N., et al. 2016, A\&A, 594, A13

Pourtsidou, A., \& Tram, T. 2016, PhRvD, 94, 043518

Raveri, M., Martinelli, M., Zhao, G., \& Wang, Y. 2016, arXiv:1606.06273

Riemer-Sørensen, S., \& Sem Jenssen, E. 2017, arXiv:1705.03653

Riess, A. G., Macri, L. M., Hoffmann, S. L., et al. 2016, ApJ, 826, 56

Ross, A. J., Samushia, L., Howlett, C., et al. 2015, MNRAS, 449, 835

Seehars, S., Amara, A., Refregier, A., Paranjape, A., \& Akeret, J. 2014, PhRvD, 90, 023533

Seehars, S., Grandis, S., Amara, A., \& Refregier, A. 2016, PhRvD, 93, 103507

Sola, J., Gomez-Valent, A., \& de Cruz Perez, J. 2017, arXiv:1705.06723

Verde, L., Protopapas, P., \& Jimenez, R. 2014, PDU, 5, 307

Wang, Y., Zhao, G.-B., Chuang, C.-H., et al. 2017, MNRAS, 469, 3762

Wang, Y., Zhao, G.-B., Wands, D., Pogosian, L., \& Crittenden, R. G. 2015, PhRvD, 92, 103005

Wyman, M., Rudd, D. H., Vanderveld, R. A., \& Hu, W. 2014, PhRvL, 112, 051302

Zhao, G.-B., Raveri, M., Pogosian, L., et al. 2017a, NatAs, 1, 627

Zhao, G.-B., Wang, Y., Ross, A. J., et al. 2016, MNRAS, 457, 2377

Zhao, G.-B., Wang, Y., Saito, S., et al. 2017b, MNRAS, 466, 762 